\section{Diagnostic evaluation of the knee}

M. Strobel and H.-W. Stedtfield

Berlin, Germany: Springer Verlag, 1990: 356pp, DM 268

This is a glossy, extensively illustrated book entirely devoted to the diagnosis and evaluation of knee injuries. Treatment is only occasionally mentioned. Originally published in German by Springer, it has been translated into English and is given an enthusiastic foreword from John Feagin, author of The Crucial Ligaments.

The book is divided into four main sections. The first on anatomy, proprioception and biomechanics is indeed impressive. The illustrations are clear and relevant with anatomical structures precisely displayed. The section on proprioception contains some useful therapeutic implications of arthrotomy. Biomechanics of the knee joint are not simple, yet the text gives a clear explanation.

The chapters on general clinical diagnosis go thoroughly through the salient points which need to be obtained from the history and examination. The clinical pictures are first class. There are tables to act as summaries, and bold print is used to stress important statements. There are some serious omissions, the importance of a block to extension is not emphasized, there is no mention of the hip as a source of knee pain in children in a paragraph devoted to that topic, and I do not think that the patella tap is the best means of detecting an effusion.

Special clinical diagnosis includes the same all-embracing care to include virtually every test for ligament laxity ever described, with appropriate advice to learn and use only those you find most useful. The explanation of the mechanism of instability is excellent. Twenty meniscus tests are described, occupying the same space as the entire section on magnetic resonance imaging. I would question the relative importance given to meniscus tests - Apley himself says he no longer uses his grinding test.

Special diagnostic procedures are described, including sections on radio- graphic examination, magnetic resonance imaging, ultrasonography, and machine evaluation of laxity, the latter giving an honest account of their worth. No description is given of means of testing the muscles controlling the knee, which are important in evaluating the function of the joint. Diagnostic arthroscopy is well covered with the usual standard of highquality pictures. A more comprehensive treatment of magnetic resonance imaging would be welcome.

This book gives a comprehensive, well illustrated account of the diagnosis and evaluation of the knee joint. It is expensive, but anyone intending to treat knees seriously needs to know the vast majority of the information given, and this book is an excellent source of reference.

\section{R. G. Hackney FRCS, Dip Sports Med}

\section{Sports Medicine for Coaches and Trainers (2nd edn)}

E. J. Shahady and M. J. Petrizzi (Eds) Chapel Hill and London: The University of North Carolina Press, 1991: 192pp

The stated aim of this book is to provide a text which gives clear guidelines for the lay teacher/trainer in the recognition and treatment of common sports injuries. There is certainly a need for a book which does not require any medical background on the part of the reader, but the task the authors have set themselves is immense.

The book is of almost A4 size, and divided into four sections. The first attempts to describe basic medical language and outlines the principles of history, examination, and the basics of treatment and rehabilitation. The second deals with specific regions of the body, and the third a wide range of related health issues such as general medical problems, nutrition, psychology and women in sport. The last section is entitled administrative issues, but includes an interesting chapter on 'How to choose a team

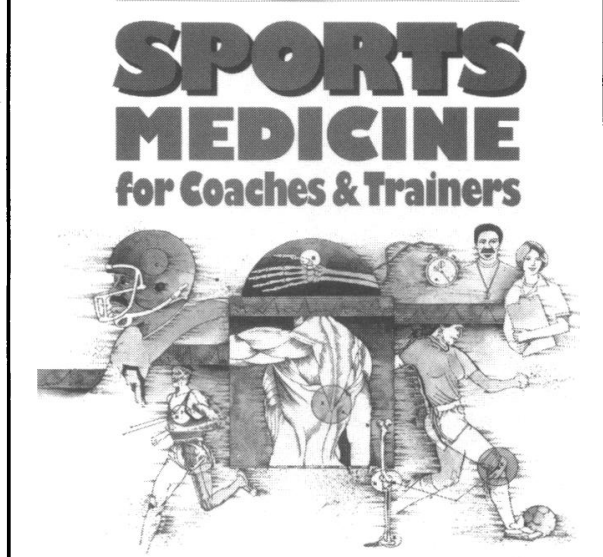

EAward H, Shahady, M.b." \& Michael J. Petrixzi, M.D.

Physician'! The many illustrations are black and white line drawings.

The nature of the readership of the book means that the explanations have to be concise, but there are many minor inaccuracies in the text and drawings, which I found irritating and may cause confusion. For example the site of a spondylolysis shown is incorrect, a blow to the front of the knee is said to cause anterior cruciate ligament tears and a separate entity called a sore arm.

Each section dealing with injuries ends with a series of exercises for recovery, but the importance of rehabilitating the athlete through to competition is not emphasized. In a chapter entitled the principles of rehabilitation, print occupies less than a page of the two allocated.

I am disappointed that there is virtually no mention of adjusting sporting technique in the management of overuse injuries. There is the briefest mention of running shoes but nothing on throwing technique or racquet use. The sports coach can contribute to the management of an injury with knowledge a doctor may not possess.

The book is readable and easy to use, and with some improvements the authors will go a long way to achieving their aims.

\section{R. Hackney FRCS, Dip Sports Med}

